\title{
Impact de la lourdeur des lourdes tâches administratives sur les soins aux patients et la satisfaction des chirurgiens
}

Les opinions exprimées dans cet éditorial sont celles de l'auteur et ne représentent pas nécessairement celles de l'Association médicale canadienne ou ses filiales.

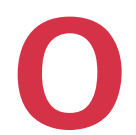

$\mathrm{n}$ peut définir les tâches administratives comme un « travail routinier impliquant des documents écrits, tels que formulaires, dossiers ou lettres ». Il est indéniable qu'une documentation adéquate (en version papier et/ou électronique) est cruciale pour la sécurité des patients, la continuité des soins et l'amélioration de leur qualité à l'échelle des individus et des systèmes ${ }^{1}$. Or, malheureusement, il semble que nos vies de chirurgiens soient de plus en plus entravées par des tâches administratives qui nous éloignent toujours plus des soins de qualité au chevet de nos patients, et nous transforment en d'efficaces scribes occupés à relater des événements médicaux passés.

La transition vers le dossier médical électronique (DMÉ) a représenté à la fois une bénédiction et une malédiction (en date de 2015, 92\% des médecins pratiquant dans les hôpitaux utilisaient les DMÉ1). Bien sûr, le DMÉ est l'avenir de notre discipline. Il permet une interface avec l'intelligence artificielle que seule la science-fiction aurait pu prédire encore récemment. Il améliore la qualité des soins, la précision diagnostique, la sécurité des patients, la continuité des soins, ainsi que le suivi des résultats et du rendement. Entre autres exemples positifs, le DMÉ facilite la gestion du flux de patients et la facturation/ rémunération en réduisant le double emploi et les retards. Par contre, ces atouts ont eu des répercussions négatives imprévues, comme l'allongement du temps de travail des chirurgiens, leur frustration face aux changements incessants des systèmes informatiques et une diminution du temps disponible pour se consacrer à leurs patients. Ce « fardeau administratif » est associé à une hausse du nombre d'erreurs médicales et d'erreurs dans la tenue des dossiers; il menace la sécurité des patients, conduit à l'attrition et cause une détresse émotionnelle chez les professionnels de la santé1. En songeant que les médecins passent à présent jusqu'à 2 fois plus de temps à remplir des formulaires électroniques et à exécuter des tâches administratives $(50 \%)$ qu'ils en passent au chevet de leurs patients (12\%-27\%), il n'est pas surprenant que l'insatisfaction des chirurgiens ait atteint un sommet inégalée-4. À notre connaissance, personne n'entreprend des études de médecine pour devenir expert en documentation - surtout si le ratio avec les soins aux patients est de 2:1.
On parle de fardeau lorsqu'une « tâche ou responsabilité est source de préoccupations et de difficultés ou représente un travail exigeant. » La notion de fardeau se distingue structurellement de l'épuisement professionnel $^{5}$. Etant donné l'importance accordée récemment au bien-être mental et physique des médecins, les chirurgiens doivent s'arrêter pour réfléchir au fardeau administratif qui leur incombe (compte tenu de leur environnement professionnel immédiat et du système de santé en place). L'évaluation objective de son impact potentiel sur le risque d'épuisement professionnel et sur la qualité de vie est un exercice nécessaire.

On songe généralement au fardeau administratif associé aux soins cliniques, mais on assiste également à une multiplication des tâches obligatoires afférentes à d'autres domaines dans plusieurs de nos pratiques. Mentionnons entre autres la recherche (comités et examens éthiques dans les universités; évaluations de l'impact de la recherche médicale à l'échelle des provinces; obstacles et freins aux programmes individuels; demandes de subventions), la rémunération (documents de facturation; confirmations des heures consignées; soumission des honoraires; facturation pro forma), l'enseignement (évaluation de la formation fondée sur les compétences; évaluation des résidents), formation professionnelle (vérifications par les instances de réglementation, évaluation des compétences et examens de maintien du certificat; sondages auprès des professionnels) et l'amélioration de la qualité des soins (intégration des nouvelles technologies et nouveaux processus, cheminements, listes de vérification, protocoles de sécurité et programmes; évaluation des incidents de sécurité). Même si ces exemples ne sont pas tous aussi chronophages ou énergivores les uns que les autres dans la pratique d'un chirurgien, ils ont tous un lien commun : l'absence de contact direct entre le médecin et son patient. Cela s'applique aussi à la formation de nos étudiants en médecine et de nos résidents, puisque l'enseignement au chevet, qui mobilisait $75 \%$ du temps de formation auparavant, n'en mobilise plus aujourd'hui qu'entre $8 \%$ et $19 \%$ selon les facultés de médecine ${ }^{1-4}$. Si plusieurs d'entre nous qui avons effectué une partie de notre formation (et/ou de notre carrière) aux États-Unis avons été sidérés par le temps consacré 
par les chirurgiens facultaires aux tâches administratives (version papier et électronique; p. ex., notes d'évolution formatées quotidiennes, dossiers d'admission, résumés de congé, mises à jour pour les assureurs, ajustements des codes de facturation), nous nous sommes tous dit « Dieu merci, le système n'est pas aussi lourd au Canada ». Il faut se rendre à l'évidence, nous sommes rendus là. En y réfléchissant bien, nos pratiques sont devenues très similaires pour ce qui est du fardeau administratif.

Fait intéressant, nous pouvons aussi nous inspirer de nos collègues du Sud de la frontière pour des solutions potentielles. Dans certains domaines de surspécialisation chirurgicale à profil élevé, nos collègues américains se sont dotés d'équipes qui s'acquittent de la grande majorité des tâches administratives. Ces pratiques chirurgicales incluent fréquemment une forme d'aide clinique non médicale dans un rapport de 1:1 pour les chirurgiens ayant des UVR (unités de valeur relatives) élevées (c.-à-d., pratiques achalandées). Les autres professionnels de la santé et périsoignants (personnel infirmier, adjoints aux médecins, associés cliniques, hospitalistes chirurgicaux) contribuent non seulement aux soins directs aux patients, mais également aux tâches administratives. Si notre réaction initiale face à un tel environnement de travail en est une d'envie, nous devrions savoir que ces ressources sont en fait déjà utilisées dans des modèles de pratique au Canada. Les exemples les plus frappants concernent nos collègues de médecine (qui sont souvent salariés, plutôt que rémunérés à l'acte). En chirurgie, plusieurs cliniques externes d'ophtalmologie achalandées utilisent des copistes, des spécialistes de la documentation et de la facturation (publics et privés) et d'autres spécialistes cliniques (p. ex., des optométristes) pour accélérer le roulement, améliorer l'efficience et favoriser davantage le lien direct chirurgien- ou médecin-patient. Cela exige sans contredit une transformation significative de nos modèles de pratique, de nos sources de financement et de notre cadre conceptuel, mais peut-être est-ce inévitable pour améliorer la satisfaction des futures générations de chirurgiens. Nous devons toutefois nous souvenir qu'au bout du compte, c'est le sentiment du devoir accompli auprès de nos patients qui est fondamental pour la réalisation de soi et la satisfaction au travail, et non la capacité de gérer des tonnes de courriels ou le fait de n'avoir aucun dossier médical incomplet.

\section{Chad G. Ball, MD, Paul B. McBeth, MD, MASc}

Affiliations: L'Université de Calgary, Calgary, Alb. (Ball); Alberta Health Services, Calgary, Alb. (McBeth); et co-éditeurr-en-chef, fournal canadien de chirurgie (Ball).

Intérêts concurrents : Aucun déclaré.

Propriété intellectuelle du contenu : Il s'agit d'un article en libre accès distribué conformément aux modalités de la licence Creative Commons Attribution (CC BY-NC-ND 4.0), qui permet l'utilisation, la diffusion et la reproduction dans tout médium à la condition que la publication originale soit adéquatement citée, que l'utilisation se fasse à des fins non commerciales (c.-à-d., recherche ou éducation) et qu'aucune modification ni adaptation n'y soit apportée. Voir : https:// creativecommons.org/licenses/by-nc-nd/4.0/.

DOI: 10.1503/cjs.015021

\section{Références}

1. Moy AJ, Schwartz JM, Chen R, et al. Measurement of clinical documentation burden among physicians and nurses using electronic health records: a scoping review. 7 Am Med Inform Assoc 2021;28:998-1008.

2. Poissant L, Pereira J, Tamblyn R, et al. The impact of electronic health records on time efficiency of physicians and nurses: a systematic review. 7 Am Med Inform Assoc 2005;12:505-16.

3. Colicchio TK, Cimino JJ. Clinicians' reasoning as reflected in electronic clinical note-entry and reading/retrieval: a systematic review and qualitative synthesis. 7 Am Med Inform Assoc 2019;26:172-84.

4. Grant P. Physician job satisfaction in New Zealand versus the United Kingdom. NZ Med 7 2004;117:U1123.

5. Jane Lemaire on burnout, resiliency and coping during COVID. Cold Steel [baladodiffusion]. Disponible ici : https://soundcloud. com/cjs-podcast/e19-jane-lemaire-on-burnout-resiliency-and -coping-during-covid (consulté le 6 mai 2021). 